\title{
Reação de cultivares de algodoeiro a Ramularia areola
}

\author{
Lonjoré Leocádio de Lima ${ }^{1,2}$, Modesto Barretoํㅜ Érika Auxiliadora Giacheto Scaloppi ${ }^{3}$
}

${ }^{1}$ Departamento de Fitossanidade, Faculdade de Ciências Agrárias e Veterinárias/ Universidade Estadual Paulista, Via de Acesso. Prof. Paulo Donato Castellane s/n, 14.884-900, Jaboticabal, SP, Brasil; ${ }^{2}$ Aluna de Pós - graduação Bolsista Capes. ${ }^{3}$ APTA. PRDTA Centro Leste, Av Bandeirantes, 2419, 14030-670, Ribeirão Preto-SP.

Autor para correspondência: Lonjoré Leocádio de Lima (lonjore@ hotmail.com)

Data de chegada: 10/07/2007. Aceito para publicação em: 29/09/2009.

\section{RESUMO}

Lima, L.L.; Barreto, M.; Giacheto Scaloppi, É.A.G. Reação de cultivares de algodoeiro a Ramularia areola. Summa Phytopathologica, v.36, n.1, p.57-60, 2010.

Dentre as doenças causadoras de manchas foliares em algodoeiro, a mancha de ramulária (Ramularia areola Atk) tem se tornado importante em virtude das condições climáticas favoráveis para o desenvolvimento do patógeno, aliadas ao uso de cultivares suscetíveis, plantio consecutivo e extenso. Neste trabalho avaliaram-se os cultivares Delta Opal, Acala 90, Makina, Delta Penta e Sure Grow 821 quanto à resistência a $R$. areola, em condições de campo na FCAV-UNESP, no ano de 2006. A severidade da doença, que ocorreu por infecção natural das plantas pelo fungo, foi avaliada semanalmente em trinta plantas devidamente marcadas, utilizando-se uma escala descritiva de notas: $1=0 \%, 2=$ até $5 \%, 3=$ de 5,1 a $25 \%, 4=$ de 25,1 a $50 \%$ e $5=$ acima de $50 \%$ de área foliar com sintomas. Foram elaboradas curvas de progresso da doença para os cinco cultivares e o modelo monomolecular foi o que melhor se ajustou aos dados, em comparação com o logístico, o exponencial e o modelo de Gompertz. A análise dos dados indicou que houve diferença significativa entre os genótipos, sendo que Delta Opal, Makina e Sure Grow 821 mostraram-se mais suscetíveis e Delta Penta e Acala 90 mais resistentes ao fungo.

Palavras-chave adicionais: Chaves Adicionais: Gossypium hirsutum, Resistência de Cultivares, Curvas de Progresso de Doenças.

\section{ABSTRACT}

Lima, L.L.; Barreto, M.; Giacheto Scaloppi, É.A.G. Cotton cultivars resistance to Ramularia areola. Summa Phytopathologica, v.36, n.1, p.5760, 2010.

Among the disease causing leaf spot in cotton, the gray mildew (Ramularia areola Atk.) has become important due to environmental conditions favorable for development of the disease susceptible cultivars, consecutive and intensive and extensive cultivation. This work evaluated the resistance of the cultivars Delta Opal, Acala 90, Makina, Delta Penta and Sure Grow 821 to resistance the $R$. areola, in field conditions in FCAV-UNESP. The disease occurred under natural infection and, its severity was evoluated weekly, in thirty previously marked plants using the following rating scale: $1=0 \%, 2=$ up to $5 \%, 3=5,1$ to $25 \%, 4=$ 25,1 to $50 \%$ and $5=$ over of $50 \%$ of foliar area with symptoms. The monomolecular model was better adjusted to the data in comparison with the logistic, exponential and Gompertz models, for elaboration of the curves of progress of the disease. The analysis of the data indicated significant differences between the genotypes.The cultivars Delta Opal, Makina and Sure Grow 821 were more susceptible and Delta Penta and Deltapine Acala 90 more resistant to gray mildew.

Keywords: Gossypium hirsutum, Cultivars reaction, Curves of progress of diseases.

A cultura do algodão (Gossypium hirsutum L.) no Brasil se estende praticamente por duas regiões: a região meridional, que compreende os estados de São Paulo, Paraná, Mato Grosso, Mato Grosso do Sul, Goiás, Minas Gerais e Sul da Bahia, onde se planta o algodão herbáceo com excelente produtividade e a região setentrional onde se localizam os estados produtores do Norte e Nordeste, caracterizada pelo cultivo tanto do algodão herbáceo como arbóreo (7).

Nas principais áreas produtoras de algodão, dentre as doenças causadoras de manchas foliares, a mancha de ramulária tem se tornado importante em virtude das condições climáticas favoráveis para o desenvolvimento do patógeno, aliadas ao uso de cultivares suscetíveis (11).

Caracteriza-se por apresentar manchas de forma angular de 1 a 4 $\mathrm{mm}$, delimitadas pelas nervuras. $\mathrm{Na}$ face inferior das folhas aparecem estruturas reprodutivas do fungo, inicialmente brancas e depois amarelas. Na face superior das folhas, em correspondência à lesão na face inferior, aparecem manchas que inicialmente são de cor verdeamarelada e que depois se tornam avermelhadas (9). O patógeno é favorecido por condições de alta umidade, temperatura entre 17 a 28 ${ }^{\circ} \mathrm{C}$ e cultivares suscetíveis (15).

A mancha de Ramulária é uma das principais doenças da cultura do algodoeiro em países como Madagascar e Índia sendo, neste último, em algumas safras responsável por perdas acima de $60 \%$ da produção $(8,16)$. No Brasil, até algum tempo atrás a mancha de Ramulária era considerada um problema fitossanitário secundário, ocorrendo apenas no final do ciclo da cultura do algodoeiro, mas nos últimos anos, com o aumento da área cultivada de algodão no cerrado e o uso de cultivares suscetíveis, a doença passou a surgir mais cedo sendo, considerada, 
hoje, a principal enfermidade da cultura nesta região, com perdas estimadas em $30 \%$ da produção (18).

Segundo Bergamin Filho (3) as curvas de progresso de doenças, usualmente são expressas pela plotagem da proporção de doença versus tempo, e é a melhor forma de apresentação de uma epidemia. Através delas, interações entre patógeno, hospedeiro e ambiente podem ser caracterizadas, estratégias de controle avaliadas, níveis futuros de doença previstos e simuladores verificados.

Independentemente da situação a ser considerada, inúmeras informações importantes podem ser obtidas a partir da análise da curva de progresso da doença, dentre as quais se destacam época e início da epidemia, quantidade de inóculo inicial, taxa de progresso da doença, intensidade máxima ou ponto de máxima da doença, intensidade final da doença, forma da curva de progresso e a duração da epidemia $(3,6,12)$. A comparação de curvas de progresso de doenças em cultivares com diferentes níveis de resistência tem sido um dos objetivos de estudo da epidemiologia comparativa (17).

Objetivou-se com este trabalho avaliar a reação de cultivares de algodão à mancha de ramulária analisada através das curvas de progresso da doença.

\section{MATERIAL E MÉTODOS}

O experimento foi desenvolvido na Fazenda de Ensino, Pesquisa e Produção da Faculdade de Ciências Agrárias e Veterinárias, da Universidade Estadual Paulista, no município de Jaboticabal, no estado de São Paulo, cujas coordenadas geográficas são: latitude de $21^{\circ} 1522^{\prime \prime}$ $\mathrm{S} ; 48^{\circ} 1858^{\prime \prime}$ e altitude de $575 \mathrm{~m}$. O clima da região, segundo a classificação de Koppen, é Cwa (subtropical úmido, seco no inverno e com chuva no verão), com precipitação média anual de $1300 \mathrm{~mm}$ e temperatura média anual de $21^{\circ} \mathrm{C}$.

$\mathrm{O}$ delineamento experimental utilizado foi o de blocos casualizados (DBC), com cinco tratamentos e quatro repetições, sendo que as parcelas experimentais foram constituídas de seis linhas de cinco metros, com espaçamento de $0,90 \mathrm{~m}$ entre linhas, sendo a área útil constituída pelas quatro linhas centrais, desprezando-se $1,0 \mathrm{~m} \mathrm{em}$ cada extremidade. A semeadura foi realizada no dia 21 de Janeiro de 2006. Os tratos culturais e as aplicações de defensivos foram os recomendados para a cultura, exceto aplicações de fungicidas, que não foram realizadas. Avaliou-se a reação de cinco cultivares de algodão (Delta Opal, Deltapine Acala 90, Makina, Delta Penta e Sure Grow 821) a Ramularia areola, durante o ano de 2006.

A severidade da doença, que ocorreu por infecção natural das plantas pelo fungo, foi avaliada semanalmente, a partir do início dos primeiros sintomas, em trinta plantas previamente marcadas, utilizando-se uma escala descritiva de notas (1):

Nota 1 - Plantas sem sintomas;

Nota 2 - Até $5 \%$ de área foliar com sintomas;

Nota 3 - De $5,1 \%$ a $25 \%$ de área foliar com sintomas;

Nota 4 - De $25,1 \%$ a 50\% de área foliar com sintomas, com queda das folhas axilares no terço médio, início de desfolha precoce no baixeiro e lesões de Ramulária no ponteiro.

Nota 5 - Acima de $50 \%$ de área foliar com sintomas, com queda de folhas no terço médio e desfolha precoce.

As notas foram convertidas em porcentagens de área foliar afetada substituindo - se a nota pelo valor médio do intervalo considerado em cada nota. A seguir essas porcentagens foram transformadas em proporção $(y)$, para testar os diferentes modelos matemáticos: exponencial $[y=\ln (y)]$, monomolecular $[y=\ln [1 /(1-\mathrm{y})]]$, logístico [y $=\ln [y /(1-\mathrm{y})]]$ e de Gompertz $[y=-\ln [-\ln (\mathrm{y})]]$, conforme recomendação de Campbell \& Madden (6). Ajustaram-se os modelos de regressão linear, tendo o tempo em dias, após o aparecimento dos primeiros sintomas da mancha de ramulária como variável independente e a severidade da doença em proporção como variável dependente e a Área Abaixo da Curva de Progresso de Doenças (AACPD), com auxílio do software Microsoft Excel 2003 ${ }^{\circledR}$. A escolha do melhor modelo baseou-se no coeficiente de determinação ajustado $\left(\mathrm{R}^{* 2}\right)$, obtido da regressão linear entre os valores previstos (Variável dependente), e observados (Variável independente) por ser um parâmetro confiável. Considerou-se como o melhor modelo aquele que apresentou a maior frequência de valores mais altos de $\mathrm{R}^{* 2}$ de acordo com as recomendações de Campbell \& Madden (6).

Os dados da Área Abaixo da Curva de Progresso de Doença (AACPD) foram analisados pelo teste $\mathrm{F}$ para variância e as médias comparadas pelo teste de Tukey a $5 \%$ de probabilidade.

\section{RESULTADOS E DISCUSSÃO}

Os valores dos coeficientes de determinação ajustados $\left(\mathrm{R}^{* 2}\right)$, desvio padrão do inóculo inicial $\left(X_{0}\right)$ e desvio padrão da taxa de infecção $(r)$ para cada transformação, nas cinco cultivares, são apresentados na Tabela 1.

Com base nestes dados observou-se que o modelo monomolecular foi o que melhor se ajustou aos dados, apresentando um maior coeficiente de determinação $\left(\mathrm{R}^{* 2}\right)$, e o menor desvio padrão da taxa de infecção (r) em comparação com o logístico, o exponencial e o modelo de Gompertz, para elaboração das curvas de progresso da doença para

Tabela 1. Coeficiente de determinação $\left(\mathrm{R}^{* 2}\right)$, desvio padrão do inóculo inicial $\left(X_{0}\right)$ e desvio padrão da taxa de infecção $(r)$ após ajuste dos modelos Exponencial, Monomolecular, Logístico e de Gompertz aos dados de severidade da mancha de ramulária na cultura do algodoeiro. JaboticabalSP, 2006.

\begin{tabular}{|c|c|c|c|c|}
\hline Variedades & Modelos & $\mathbf{R} *^{2}$ & $\left(\boldsymbol{X}_{0}\right)$ & $(\boldsymbol{r})$ \\
\hline \multirow[t]{4}{*}{ Delta Penta } & Exponencial & 0,70 & $-6,5827$ & 0,0207 \\
\hline & Monomolecular & 0,81 & $-0,0074$ & 0,0002 \\
\hline & Logístico & 0,70 & $-6,5901$ & 0,0209 \\
\hline & Gompertz & 0,73 & $-1,9395$ & 0,0044 \\
\hline \multirow[t]{4}{*}{ Sure Grow 821} & Exponencial & 0,73 & $-6,7525$ & 0,0290 \\
\hline & Monomolecular & 0,86 & $-0,0380$ & 0,0006 \\
\hline & Logístico & 0,74 & $-6,7905$ & 0,0296 \\
\hline & Gompertz & 0,78 & $-2,0826$ & 0,0075 \\
\hline \multirow[t]{4}{*}{ Makina } & Exponencial & 0,72 & $-6,8110$ & 0,0296 \\
\hline & Monomolecular & 0,85 & $-0,0381$ & 0,0006 \\
\hline & Logístico & 0,72 & $-6,8491$ & 0,0302 \\
\hline & Gompertz & 0,77 & $-2,0935$ & 0,0076 \\
\hline \multirow[t]{4}{*}{ Delta Penta } & Exponencial & 0,89 & $-6,7987$ & 0,0286 \\
\hline & Monomolecular & 0,95 & $-0,0368$ & 0,0006 \\
\hline & Logístico & 0,89 & $-6,8356$ & 0,0292 \\
\hline & Gompertz & 0,92 & $-2,0878$ & 0,0073 \\
\hline \multirow[t]{4}{*}{ Acala 90} & Exponencial & 0,78 & $-7,5877$ & 0,0284 \\
\hline & Monomolecular & 0,91 & $-0,0125$ & 0,0002 \\
\hline & Logístico & 0,78 & $-7,6002$ & 0,0287 \\
\hline & Gompertz & 0,82 & $-2,1275$ & 0,0058 \\
\hline
\end{tabular}




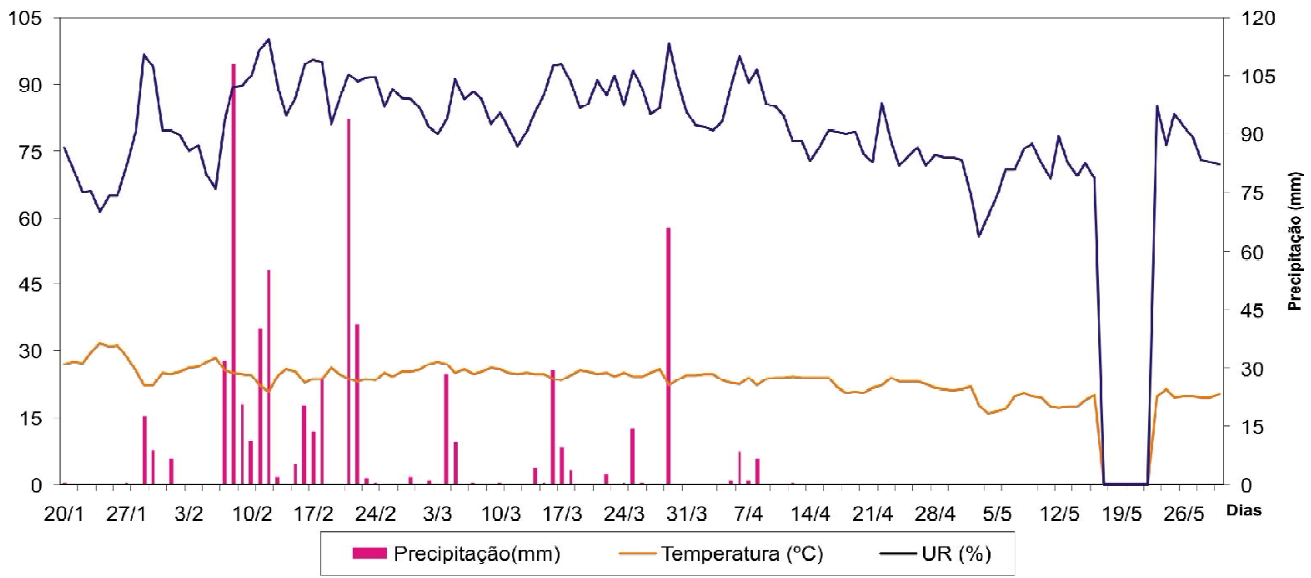

Figura 1.Variação diária dos dados de precipitação, temperatura e umidade relativa (UR) durante a avaliação do experimento. Jaboticabal-SP 2006.

as cinco cultivares, já para o desvio padrão do inóculo inicial $\left(X_{0}\right)$ o modelo que melhor se ajustou foi o logístico. Entretanto, optou-se pela a escolha baseada nos valores de $\mathrm{R}^{* 2}$ que segundo Bergamin Filho (3) é um parâmetro confiável.

Este modelo tem sido indicado para ajuste de dados de doenças com período de incubação variável (4). No modelo monomolecular, a velocidade de progresso da doença ou aumento da epidemia, expressa pelo diferencial, é proporcional ao inóculo inicial $(X o)$ e a taxa de progresso $(r)$ é constante. A velocidade de aumento $(d y / d t)$ diminui ao longo do tempo e aproxima-se de zero à medida que o tecido sadio se esgota (3). Entretanto é importante ressaltar que informações estatísticas sobre ajuste de modelos teóricos devem ser interpretadas com cautela já que as mesmas são baseadas em premissas que nem sempre ocorrem na natureza $(3,6)$.

Apesar do excelente resultado do modelo monomolecular, a mancha de ramulária como a maioria das doenças, sofre bastante influência das condições climáticas, principalmente o período de molhamento foliar, temperatura, chuva e umidade relativa (9). Essas variáveis praticamente se mantiveram constantes durante todo o período de avaliação do experimento com exceção da precipitação. Os dados de precipitação e umidade relativa em um determinado momento do experimento não foram coletados devidos a problemas nos equipamentos de coleta, como mostra a Figura 1.

Através da Figura 1 observa que a temperatura manteve-se constante em aproximadamente $27^{\circ} \mathrm{C}$, dentro de uma faixa ótima para o desenvolvimento do fungo que é em torno de 25 a $30^{\circ} \mathrm{C}$.

A umidade relativa ficou entre 80 a $90 \%$ excelente já que o mesmo desenvolve-se bem em ambientes úmidos e com temperaturas elevadas, as chuvas alternadas com períodos secos é de fundamental importância para a infecção do algodoeiro pelo fungo $R$. areola, já que o mesmo necessita de um período de molhamento foliar alternado de secamento conforme Rathaiah (14).

Rathaiah (14), estudando a penetração de $R$. areola em diferentes temperaturas de 16 a $30^{\circ} \mathrm{C}$ tanto em condições de casa de vegetação como em laboratório, observou que o mesmo apresentou melhor germinação nas temperaturas de $25-30^{\circ} \mathrm{C}$ e a maior penetração do fungo ocorreu sob o ciclo de molhamento noturno e secamento diurno do que em molhamento contínuo. Este autor também observou que algumas infecções ocorrem depois de dois ciclos de molhamento noturno de 12 horas, o máximo ocorre depois de cada quatro ciclos. Verificou ainda que o tubo germinativo pode sobreviver por vários ciclos de secamento de 16 horas com umidade relativa de 20 a $60 \%$ e subseqüentemente, retoma o crescimento.
Tabela 2. Comparação de médias da Área Abaixo da Curva de Progresso (AACPD) para mancha de Ramulária em cinco cultivares de algodoeiro. Jaboticabal-SP, 2006.

\begin{tabular}{ll}
\hline Cultivares & Médias \\
\cline { 2 - 2 } & AACPD \\
\hline Delta Penta & $709,21 \mathrm{a}$ \\
Sure Grow 821 & $1477,91 \quad \mathrm{~b}$ \\
Makina & $1485,75 \quad$ b \\
Delta Opal & $1325,06 \quad$ b \\
Acala 90 & 601,70 a \\
\hline
\end{tabular}

*Médias seguidas de mesma letra não diferem pelo teste de Tukey, a 5\% de probabilidade.

O resultado do teste de comparação de médias apresentado na Tabela 2 mostrou que houve diferença significativa entre os genótipos, sendo que Delta Opal, Makina e Sure Grow 821 mostraram-se mais suscetíveis e Delta Penta e Deltapine Acala 90 mais resistentes a $R$. areola. Durante a condução do ensaio as cultivares estudadas não apresentaram nenhuma outra doença comum a cultura do algodoeiro somente a mancha de ramulária.

Galbiere (10), testando vários genótipos em diferentes regiões no Brasil nos anos agrícolas de 2004/2005 e 2005/2006 também observou que as cultivares Delta Opal e Makina foram suscetíveis à mancha de ramulária com destaque para a Makina por apresentar altos índices da doença em locais favoráveis ao desenvolvimento do patógeno.

Pilizel et al (13), estudando dez genótipos de algodoeiro com relação a suscetibilidade a mancha de ramulária, observou que os mesmos não diferiram significativamente entre si. Desses dez genótipos dois foram utilizados nesse trabalho (Deltapine Acala 90 e Delta Opal) e os mesmo apresentaram comportamento diferente. Deltapine Acala 90 apresentou-se menos suscetível que o Delta Opal. Essa diferença pode ser devido as condições climáticas dos locais dos experimentos, potencial de inóculo e até a variabilidade do patógeno.

\section{REFERÊNCIAS BIBLIOGRÁFICAS}

1. Araújo, A.E.; Suassuna, N.D.; Freitas, J.S.; Farias F.J.C. Escala de notas para avaliação de doenças foliares do algodoeiro. In: Congresso Brasileiro de Algodão,4., 2003, Goiânia, Campina Grande: Embrapa Algodão, 2003. p. 1-7. CDROM.

2. Bedendo, I.P. Ambiente e doença. In: Bergamin Filho, A.; Kimati, H.; Amorim, L. Manual de fitopatologia. 3 ed. São Paulo: Agronômica Ceres, 1995. v.1, p. 331-341. 
3. Bergamin Filho, A. Curvas de progresso da doença. In: Bergamin Filho, A; Kimati, H.; Amorim, L. Manual de fitopatologia. São Paulo: Agronômica Ceres, 1995. p. 602-626.

4. Bergamin Filho, A; Amorim, L. Doenças com período de incubação variável em função da fenologia do hospedeiro. Fitopatologia Brasileira, Brasília, v.27, p 561-565, 2002.

5. Bergamin Filho, A; Amorim, L. Doenças de plantas tropicais: epidemiologia e controle. São Paulo: Agronômica Ceres, 1996, $289 \mathrm{p}$

6. Campbell, C.L; Madden, L. V. Introduction to plant disease epidemiology. New York: Wiley - Intersiencie, 1990. 532p.

7. Carvalho, L.H; Chiavegato, E.J. A cultura do algodão no Brasil fatores que afetam a produtividade. In: Cia, E.; Freire, E.C.; Santos, W.J. Cultura do algodoeiro. Piracicaba: Potafós, 1999. p. $1-8$

8. Cauquil,J.;Sément,G. Le faux mildiou du cotonnier (Ramularia areola Atk.) dans lê sud-ouest de Madagascar. Coton et Fibres Tropicales, Paris, v.28, p.279-289, 1973.

9. Cia, E.; Salgado, C. Doenças do Algodoeiro. In: Kimati, H.; Amorim, L.; Rezende, J.A.M.; Bergamin filho, A.; Camargo, L.E.A. Doenças das plantas cultivadas, $4^{\circ}$ ed. São Paulo: Agronômica Ceres, 2005. v.2, p. 41- 42 .

10. Galbiere, R. Comportamento de genótipos de algodoeiro na presença de patógenos e nematóides. 2007. 80 f. Dissertação (Mestrado em Agricultura Tropical e Subtropical) - Instituto Agro- nômico, Campinas.

11. Iamamoto, M.M. Doenças foliares do algodoeiro. Jaboticabal: FUNEP, 2003. p. 41.

12. Kranz, J. Comparative of epidemics. Annual Review Phytophatology, Palo alto, v.12, p 355-374, 1974

13. Polizel, A.C; Juliatti, F. C; Penna, J. V; Hamawaki, O. T. Reação de genótipos de algodoeiro quanto à severidade de manchas foliares. Bioscience Journal, Uberlândia, v.24, n. 3, p.8-12. 2008.

14. Rathaiah,Y. Spore germination and mode of cotton infection by Ramularia areola. Phytopathology, St Paul, v.67, p. 351-357. 1977.

15. Shivankar, S.K.;Anvikar, D.G. Occurrence and development of grey mildew of cotton caused by Ramularia gossypii Speg. In Relation to weather. J. Soils and Crops., v. 5, n. 2, p. 153-156, 1995.

16. Shivankar, S.K.; Wangikar, P.D. Estimation of crop losses due to grey mildew disease of cotton caused by Ramularia areola. Indian Phytopathology, St Paul, v.45, n.1, p. 74-76, 1992.

17. Spósito, M.B; Bassanezi, R.B; Amorin, L. Resistência à mancha preta dos citros avaliada por curva de progresso de doença. Fitopatologia Brasileira, Brasília, v.29, p. 532 - 537, 2004.

18. Suassuna, N.D.; Iamamoto, M.M. Controle químico da mancha de Ramulária do algodoeiro. In: Congresso Brasileiro de Algodão, 5, 2005, Salvador, Anais, Campina Grande: Embrapa Algodão, 2003. p. 1-7, CDROM. 